\title{
Grey Solitons in Saturable Media with Nonlinear Dispersion and Self-Frequency Shift
}

\author{
J. JASIŃSKI ${ }^{*}$ \\ Faculty of Physics, Warsaw University of Technology \\ Koszykowa 75, 00-662 Warszawa, Poland
}

\begin{abstract}
In the paper a special form of the generalized nonlinear Schrödinger equation in saturable medium with nonlinear dispersion and the self-frequency shift terms is considered. The solutions describing both the intensity and phase profiles of grey solitons propagating in this medium are obtained. Four cases of medium with or without saturation and with or without higher order terms are considered. For a square-two-level model of saturation analytical explicit solutions are derived. The relations between the medium and propagation parameters of solitons are discussed.
\end{abstract}

PACS numbers: 42.65.Tg, 42.82.Et

\section{Introduction}

Light pulse propagating through a nonlinear Kerr-like dielectric interacts with it in many ways. The effects caused by this interaction change the shape of the pulse. Among them two are most important - the linear effect of group velocity dispersion (GVD) and self-phase modulation (SPM) caused by nonlinear polarization of the medium [1-3]. If these two effects mutually compensate, the pulse of a special shape - soliton - can travel through the medium without any changes. But for the strong electric field of the pulse also another effects should be taken into account. One of them - the third order dispersion — is linear [4-6]; the others are nonlinear. In the paper two of such effects are considered - nonlinear dispersion (ND) [6-9] and self-frequency shift (SFS) [10, 11]. The propagation of the pulse envelope taking into account these higher-order effects is described by a generalized nonlinear Schrödinger equation (GNLSE). This equation also possesses

*e-mail: jasinski@if.pw.edu.pl 
solutions in the form of solitons, but special requirements between parameters of the medium and soliton are needed in order to preserve the original profile from the pure Kerr dielectric [6, 12-16].

In the presence of strong soliton field the nonlinear permittivity of the medium can deviate from the simple Kerr dependence revealing saturation. The simplest description of this phenomenon consists in adding a small negative term to Kerr formula, which gives a cubic-quintic model of saturation [20-22]. This model enables analytical solutions of many problems involving non-Kerr media, but in fact does not predict any saturation for large intensity. The other models of saturation - two-level [17] and exponential [18] - guarantee the proper behavior of permittivity for large intensity, but practically none of the problems in such media cannot be solved analytically. The most promising is the square-two-level model [19] with a proper large intensity limit and many analytical solutions. Therefore this model of saturation will be applied in the paper. In fact, all discussed models give very similar results far below saturation, so the application of any of them is the matter of convenience.

The form of nonlinear polarization should affect all nonlinear terms in GNLSE. Therefore, introducing saturation into GNLSE, we should include it also into the higher-order terms [8, 23, 24]. The resulting GNLSE equation is possible to solve for bright and dark soliton cases [23, 24]. The obtained solutions express the intensity and phase profiles in a form of certain quadratures. In the presented paper we expand the previous solutions to include grey solitons, whose propagation in the generalized Kerr-like medium has not been analyzed yet.

\section{Quadratures of intensity and phase}

The nonlinear medium reacts to the traveling wave changing its own dielectric permittivity $\varepsilon$ by $\varepsilon_{\mathrm{NL}}$. Such response of the medium for Kerr-like materials depends on light intensity $I=|\boldsymbol{E}|^{2}$ and in a pure Kerr case is proportional to intensity

$$
\varepsilon_{\mathrm{NL}}(I)=\alpha I \text {. }
$$

If the light intensity is sufficiently strong, the nonlinear permittivity deviates from the above rule because of saturation. The square-two-level model [19] describing this process gives the modified function (1) in the following form:

$$
\varepsilon_{\mathrm{NL}}(I)=\frac{\alpha I\left(I+2 I_{\mathrm{s}}\right) I_{\mathrm{s}}}{2\left(I+I_{\mathrm{s}}\right)^{2}}
$$

where $I_{\mathrm{s}}$ is the parameter of saturation $\left(\lim _{I \rightarrow \infty} \varepsilon_{\mathrm{NL}}(I)=\alpha I_{\mathrm{s}} / 2\right)$.

According to the introduction, the non-Kerr permittivity function $\varepsilon_{\mathrm{NL}}(I)$ (2) influences two other nonlinear terms in GNLSE [8, 17, 18, 20-24]. Neglecting the linear third order dispersion effect and all processes in which energy is lost, we 
can write the GNLSE for the slowly varying envelope $U(z, t)$ of the electric field $E(z, t)$ in the frame moving with the group velocity $v_{\mathrm{g}}=\mathrm{d} \omega / \mathrm{d} k$ of the packet [23]

$$
\begin{aligned}
\frac{\partial U}{\partial z}+ & \frac{\mathrm{i} k_{2}}{2} \frac{\partial^{2} U}{\partial t^{2}}-\mathrm{i} \varepsilon_{\mathrm{NL}}\left(|U|^{2}\right) U+\frac{2}{\omega} \frac{\partial}{\partial t}\left(\varepsilon_{\mathrm{NL}}\left(|U|^{2}\right) U\right) \\
& +\tau U \frac{\partial}{\partial t} \varepsilon_{\mathrm{NL}}\left(|U|^{2}\right)=0 .
\end{aligned}
$$

In this equation $k_{2}$ determines the group velocity dispersion, $\omega$ is the carrier wave frequency and $\tau$ (real) is the retardation time.

Soliton solutions of the above equation characterize by the intensity profiles not changing during propagation. Therefore such a profile depends on one coordinate only in the frame propagating with the field envelope $I(z, t)=|U(z, t)|^{2}=$ $I(s)$. Defining $T$ and $1 / \Omega$ as temporal and spatial widths of the pulse we have

$$
s=\frac{t-k_{2} \Omega z}{T} .
$$

Let us decompose GNLSE (3) into parts using intensity of the pulse and its phase

$$
U(z, t)=\sqrt{I(s)} \exp \left[\frac{\mathrm{i} k_{2}}{2}\left(\frac{L}{T^{2}}-\Omega^{2}\right) z-\mathrm{i} \Omega T s+\mathrm{i} \varphi(s)\right] .
$$

The additional terms in phase have been introduced in order to simplify the resulting equations. The equation for the phase can be integrating twice giving [23, 24]

$$
\varphi(s)=\int_{0}^{s}\left\{\frac{2 T}{\omega k_{2}}\left[(\kappa+1) \varepsilon_{\mathrm{NL}}(I(s))-\frac{\kappa F(I(s))}{I(s)}\right]+\frac{C}{I(s)}\right\} \mathrm{d} s .
$$

In this expression $C$ is the constant of integration constants ( $L$ appearing in (5) is another such constant), $\kappa$ is a combinedhigher-order corrections parameter

$$
\kappa=1+\omega \tau,
$$

and $F(I)$ is an auxiliary function defined by means of nonlinear permittivity $\varepsilon_{\mathrm{NL}}(I)$

$$
F(I)=\int_{0}^{I} \varepsilon_{\mathrm{NL}}(I) \mathrm{d} I .
$$

The intensity function $I(s)$ satisfies the equation that follows from GNLSE if we eliminate the phase function. This equation is quite complicated and can be integrated only once. Nevertheless the solution of the resulting ordinary first-order differential equation can be written as quadrature

$$
s(I)= \pm \int_{I_{0}}^{I} \frac{\mathrm{d} I}{2 \sqrt{\frac{4 \kappa^{2} G(I)}{q \alpha^{2} I_{\mathrm{K}}^{2}}+\frac{2 p I F(I)}{\alpha I_{\mathrm{K}}}-L I^{2}+J I-C^{2}+\frac{4 C p \kappa F(I)}{\sqrt{q} \alpha I_{\mathrm{K}}}}} .
$$

In the above integral $G(I)$ is another function depending on nonlinear permittivity

$$
G(I)=\frac{\kappa^{2}-1}{\kappa^{2}} I \int_{0}^{I} \varepsilon_{\mathrm{NL}}^{2}(I) \mathrm{d} I-\left(\int_{0}^{I} \varepsilon_{\mathrm{NL}}(I) \mathrm{d} I\right)^{2},
$$


while $q$ and $I_{\mathrm{K}}$ are the temporal width and Kerr intensity parameters

$$
q=(2 \Omega+\omega)^{2} T^{2}, \quad I_{\mathrm{K}}=\frac{\omega}{(2 \Omega+\omega) T^{2}}\left|\frac{k_{2}}{\alpha}\right|,
$$

$p$ is the sign of the ratio of group velocity dispersion coefficient to the constant of Kerr nonlinearity: $p=\operatorname{sign}\left(k_{2} / \alpha\right)= \pm 1$ and $J$ is another constant of integration. The quadratures (6) and (9) generalize similar solutions for bright and dark solitons [23, 24], because the constant $C$ vanished in previous cases.

Taking into account types of media covered by solutions (6) and (9) we can distinguish four special cases. The simplest is the case of the pure Kerr medium with $\varepsilon_{\mathrm{NL}}(I)$ given by (1) and vanishing higher order terms in (3). To write the solution for this case we should take the appropriate function $F(I)$ and put $q \rightarrow \infty$ (the ND term vanishes for $\omega \rightarrow \infty$, which gives infinite $q$ ). Leaving the terms containing $q$ we arrive to the case of the generalized Kerr medium. For both these cases we have

$$
F(I)=\frac{\alpha I^{2}}{2}, \quad G(I)=\frac{\alpha^{2}\left(\kappa^{2}-4\right) I^{4}}{12 \kappa^{2}} .
$$

Another functions $F(I)$ and $G(I)$ result for the square-two-level medium. Applying $\varepsilon_{\mathrm{NL}}(I)$ given by (2) to calculate (8) and (10) we have

$$
F(I)=\frac{\alpha I^{2} I_{\mathrm{s}}}{2\left(I+I_{\mathrm{s}}\right)}, \quad G(I)=\frac{\alpha^{2} I^{4}\left[\left(\kappa^{2}-4\right) I_{\mathrm{s}}-3 I\right] I_{\mathrm{s}}^{2}}{12 \kappa^{2}\left(I+I_{\mathrm{s}}\right)^{3}} .
$$

Introducing (13) into (6) and (9) we can obtain the case of saturable medium (for $q \rightarrow \infty$ ) and the most complicated generalized saturable case.

\section{Grey solitons in Kerr and saturable media}

The result of quadrature (9) depends on three integration constants $L, C$, and $J$ and the central intensity $I_{0}=I(0)$. Their values should be established by behavior of the solution at $s=0$ and for $s \rightarrow \pm \infty$, which depends on the type of propagating soliton. Bright soliton [23] is obtained when $L=-1, C=0$, $J=0$ with $I_{0}$ remaining free, dark soliton [24] results when $C=0$ and $I_{0}=0$, while integral (9) with all non-vanishing terms describes grey soliton. In this case two of these constants are fixed and two other remain free. But the constants of integrations have no simple interpretation, so instead of them let us introduce two more natural quantities characterizing soliton - the background level $I_{\mathrm{b}}$ and the darkness $0 \leq w \leq 1$

$$
I_{\mathrm{b}}=\lim _{s \rightarrow \pm \infty} I(s), \quad w=1-\frac{I_{0}}{I_{\mathrm{b}}} .
$$

In the pure Kerr case grey soliton solutions exist only for positive medium sign coefficient $p=\operatorname{sign}\left(k_{2} / \alpha\right)=+1$. Both integrations (9) and (6) can be easily

performed giving 


$$
\begin{aligned}
& I(s)=I_{\mathrm{b}}\left(1-\frac{w}{\cosh ^{2} s}\right), \\
& \varphi(s)=\sqrt{\frac{1-w}{w}} s+\arctan \left(\sqrt{\frac{w}{1-w}} \tanh s\right) .
\end{aligned}
$$

For the above solution we have $\Omega=0, w=I_{\mathrm{K}} / I_{\mathrm{b}}$ and $L=3 / w-1$. Such intensity profile $I(s)$ for $w=1 / 4$ is illustrated by the dotted line in Fig. 1. The quite simple form of the phase $\varphi(s)$ enables us to write the full envelope $U(z, t)$

$$
U(z, t)=\sqrt{I_{\mathrm{b}}}(\sqrt{1-w}+\mathrm{i} \sqrt{w} \tanh (t / T)) \exp \left(\frac{\mathrm{i} k_{2} L z}{2 T^{2}}+\frac{\mathrm{i} \sqrt{1-w}}{\sqrt{w}} \frac{t}{T}\right)
$$

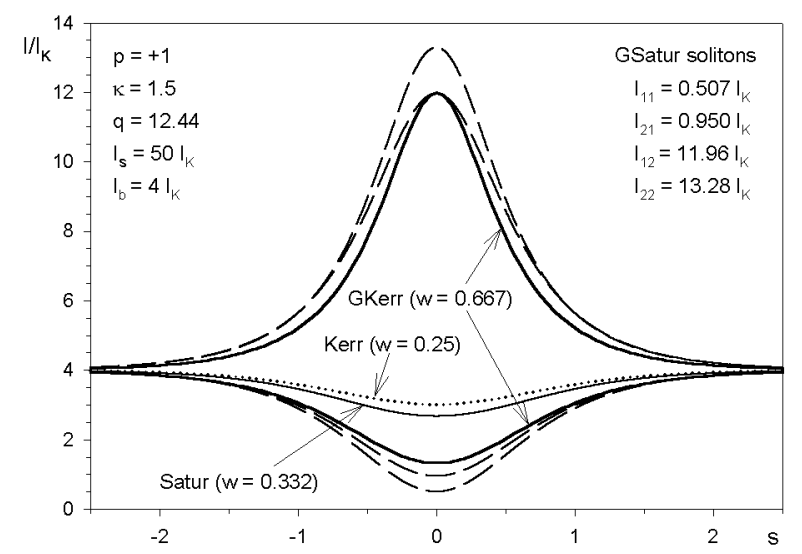

Fig. 1. Intensity profiles of the grey solitons in Kerr, generalized Kerr, saturable, and generalized saturable media. Two values of integration constant $C$ in GSatur medium determine two dark-like solitons with central intensity $I_{11}$ and $I_{21}$ and two bright-like solitons with central intensity $I_{12}$ and $I_{22}$ (dashed curves).

The solution in the generalized Kerr medium is a bit more complicated. In this case grey solitons can appear not only for positive sign of $k_{2} / \alpha$, but also for the negative sign case: $p=+1$ or $p=-1$. Moreover we can obtain two types of grey solitons - normal dark-like with $0 \leq I_{0} \leq I_{\mathrm{b}}$ and upturned bright-like ones with $I_{0} \geq I_{\mathrm{b}}$. For both these types the background level $I_{\mathrm{b}}$ and darkness $w$ are the same, but their intensity and phase profiles are described by different functions

$$
\begin{aligned}
I(s) & =I_{\mathrm{b}}\left(1-\frac{w}{\cosh ^{2} s+\sigma \sinh ^{2} s}\right), \\
\varphi(s) & =\frac{\sqrt{(1-w)(\sigma+w)} s}{w}+\arctan \left(\sqrt{\frac{\sigma+w}{1-w}} \tanh s\right) \\
& +\frac{(\kappa+2)[w+\sigma(4-w)]}{\sqrt{q} \omega}\left[\frac{s}{w}-\frac{\arctan (\sqrt{\sigma} \tanh s)}{\sqrt{\sigma}}\right],
\end{aligned}
$$


for the dark-like grey solitons, while for the bright-like case we have and

$$
\begin{aligned}
I(s) & =I_{\mathrm{b}}\left(1+\frac{w}{\sigma \cosh ^{2} s+\sinh ^{2} s}\right), \\
\varphi(s) & =\frac{\sqrt{(1-w)(\sigma+w)} s}{w}-\arctan \left(\sqrt{\frac{1-w}{\sigma+w}} \tanh s\right) \\
& +\frac{(\kappa+2)[w+\sigma(4-w)]}{\sqrt{q} w \sqrt{\sigma}}\left[\frac{\sqrt{\sigma} s}{w}+\arctan \left(\frac{\tanh s}{\sqrt{\sigma}}\right)\right] .
\end{aligned}
$$

In the above formulae $\sigma$ is the additional broadening parameter. Both darkness $w$ and broadening $\sigma$ depend on material parameters: $w=\sqrt{(2-p \lambda)^{2}+2 \lambda I_{\mathrm{K}} / I_{\mathrm{b}}}+$ $2-p \lambda, \sigma=w^{2} I_{\mathrm{b}} /\left(2 \lambda I_{\mathrm{K}}\right)$, where $\lambda=3 q I_{\mathrm{K}} /\left[2\left(4-\kappa^{2}\right) I_{\mathrm{b}}\right]$. Thick solid lines in Fig. 1 illustrate intensity profiles of these two solitons. As we can see, the general shape of these profiles are similar to the profile in the pure Kerr medium, however for the assumed choice of the medium parameters their central intensities differ quite significantly.

The functions (2) and (13) describing the saturable square-two-level medium also enable us to perform integration (9) and (6) analytically. But even if higher order terms in GNLSE (3) are rejected, the resulting function $s(I)$, however quite simple, will be irreversible. Consequently, the phase cannot be expressed as an explicit function of normalized time coordinate $s$, but as a function of intensity

$$
\begin{aligned}
\pm s= & \operatorname{arctanh} \sqrt{\frac{\left(I-I_{0}\right)\left(I_{\mathrm{s}}+I_{\mathrm{b}}\right)}{\left(I+I_{\mathrm{s}}\right)\left(I_{\mathrm{b}}-I_{0}\right)}}-\sqrt{\frac{I_{\mathrm{b}}-I_{0}}{I_{\mathrm{b}}+I_{\mathrm{s}}} \operatorname{arctanh} \sqrt{\frac{I-I_{0}}{I+I_{\mathrm{s}}}}}, \\
\varphi(I)= & \sqrt{\frac{I_{0}\left(I_{\mathrm{s}}+I_{\mathrm{b}}\right)}{I_{\mathrm{s}}\left(I_{\mathrm{b}}-I_{0}\right)}} \operatorname{arctanh} \sqrt{\frac{\left(I-I_{0}\right)\left(I_{\mathrm{s}}+I_{\mathrm{b}}\right)}{\left(I+I_{\mathrm{s}}\right)\left(I_{\mathrm{b}}-I_{0}\right)}} \\
& +\operatorname{arctanh} \sqrt{\frac{I_{\mathrm{s}}\left(I-I_{0}\right)}{I_{0}\left(I+I_{\mathrm{s}}\right)}},
\end{aligned}
$$

where the central value of intensity is $I_{0}=I_{\mathrm{s}}{ }^{4}\left(I_{\mathrm{s}}+I_{\mathrm{b}}\right) /\left[I_{\mathrm{s}}^{4}+I_{\mathrm{K}}\left(I_{\mathrm{s}}+I_{\mathrm{b}}\right)^{3}\right]-I_{\mathrm{s}}$.

Nevertheless we cannot reverse the intensity profile function $s(I)$, it can be plotted without any problems. This profile is given by the thin solid line in Fig. 1. It is very similar to the profile in the pure Kerr medium, however saturation causes intensity of smaller values. The similarity in shape means that the functions (19) and (17) with properly tuned $w$ and $\sigma$ can be close. Indeed, for $I_{\mathrm{s}} \rightarrow \infty$ the last term in the function $s(I)$ given by (19) vanishes and we can treat it as a small perturbation. This interpretation enables us to include it inside the first arctanh function. In this way we can reverse $s(I)$ obtaining $I(s)$ exactly in the form (17) with $w=1-I_{0} / I_{\mathrm{b}}$ and $\sigma=\left(I_{\mathrm{b}}-I_{0}\right) / I_{\mathrm{s}}$. As in the pure Kerr medium, grey soliton exists only for positive sign of $k_{2} / \alpha$. 
The most complicated is the case of generalized saturable medium, but for the square-two-level saturation the integrations (9) and (6) are also possible to perform. The resulting intensity profiles $s(I)$ in the dark-like and bright-like soliton cases will be expressed by a combination of the elliptic integral of the first and third kind [25]

$$
\begin{aligned}
& \pm s=A_{\mathrm{d}}\left[r_{0} F\left(\theta_{\mathrm{d}}(I), m\right)-\Pi\left(\theta_{\mathrm{d}}(I), \lambda_{\mathrm{d} 1}, m\right)+r_{\mathrm{d}} \Pi\left(\theta_{\mathrm{d}}(I), \lambda_{\mathrm{d} 2}, m\right)\right] \\
& \pm s=A_{\mathrm{b}}\left[\Pi\left(\theta_{\mathrm{b}}(I),-\lambda_{\mathrm{b} 1}, m\right)+r_{\mathrm{b}} \Pi\left(\theta_{\mathrm{b}}(I), \lambda_{\mathrm{b} 2}, m\right)\right] .
\end{aligned}
$$

In the above formulae $\theta_{\mathrm{b}}(I)$ for bright-like and $\theta_{\mathrm{d}}(I)$ for dark-like soliton case are two functions of intensity, while $0 \leq m \leq 1$ is the parameter of the elliptic functions. They are defined by means of $I_{\mathrm{s}}, I_{\mathrm{b}}$ and $I_{0}, I_{1}$, and $I_{2}$ being roots of a certain third-order algebraic equation

$$
\begin{aligned}
& \theta_{\mathrm{d}}(I)=\arcsin \sqrt{\frac{\left(I-I_{0}\right)\left(I_{1}+I_{2}\right)}{\left(I+I_{2}\right)\left(I_{1}-I_{0}\right)}}, \quad \theta_{\mathrm{b}}(I)=\arcsin \sqrt{\frac{\left(I_{1}-I\right)\left(I_{0}+I_{\mathrm{s}}\right)}{\left(I+I_{\mathrm{s}}\right)\left(I_{1}-I_{0}\right)}} \\
& m=\sqrt{\frac{\left(I_{1}-I_{0}\right)\left(I_{\mathrm{s}}-I_{2}\right)}{\left(I_{0}+I_{\mathrm{s}}\right)\left(I_{1}+I_{2}\right)}}
\end{aligned}
$$

The other coefficients $A_{\mathrm{d}}, A_{\mathrm{b}}, r_{0}, r_{\mathrm{d}}, r_{\mathrm{b}}, \lambda_{\mathrm{d} 1}, \lambda_{\mathrm{d} 2}, \lambda_{\mathrm{b} 1}$, and $\lambda_{\mathrm{b} 2}$ in (20) are given by square roots similar to those in (21). The quantity $I_{0}$ and $I_{1}$ appearing in all these formulae are central intensity $I(0)$ of the dark-like and bright-like grey solitons.

Note that the constant of integration $C$ appears in quadrature (9) in two terms - one containing $C^{2}$ and the other with $C$. Therefore trying to determine $C$ we obtain two different values. Consequently, all quantities calculated later $I_{0}, I_{1}$, $m$ and so on, will be double! These double solutions corresponding to both dark-like and bright-like solitons can be seen in Fig. 1 (dashed lines). All these lines are similar to thick solid lines corresponding to solutions in generalized Kerr medium, however we can observe that solitons in saturable medium are a bit wider. Also a possibility to appear in media with $p=-1$ is exactly the same as in the unsaturable case. But unlikely to that case, the profiles (20) cannot be reversed in the limit $I_{\mathrm{s}} \rightarrow \infty$.

The phase quadrature (6) are also possible to integrate analytically, but the result can be expressed only as a function of intensity $\varphi=\varphi(I)$, analogously to (19). This result has a similar form to expressions (20), however contains one more elliptic function of the third kind $\Pi(\theta(I), \lambda, m)$ in each formula. Note that for two cases without saturation $\varphi(s)$ contains a term linearly changing with $s$ (see (15), (17), and (18)). The similar term also will appear in (19) if we eliminate the first term of $\varphi(I)$ using $s(I)$. Analogously we can prove that such linear term appears also in the result of integration (6) for the generalized saturable case. Therefore the phases of different solutions linearly diverge for $s \rightarrow \pm \infty$. Consequently, phase profiles can differ significantly, even if solutions are close. 


\section{Parameters of grey solitons}

The solitons obtained in the previous section have parameters being functions of a certain number of another quantities $p, I_{\mathrm{K}}, q, \kappa, I_{\mathrm{s}}$, and $I_{\mathrm{b}}$. Depending on the type of medium, certain of these quantities can or cannot influence the soliton parameters. Most of the relations between parameters in three simpler cases have been reported throughout the paper, however their full discussion in all possible regimes of values is very tedious. But in the generalized saturable case, however we are able to write the relations between parameters in the form of polynomial equations, the degree of resulting polynomials is quite high, so such formulae will be very difficult to analyze. Therefore we shall discuss such relations only using their graphical representation.

In Fig. 1 we observe the differences in central values of intensity in different types of media. These differences change with the level of saturation $I_{\mathrm{s}}$, which was illustrated in Fig. 2. Naturally, the solitons in saturable media approach Kerr solitons with increasing saturation intensity $I_{\mathrm{s}}$ both in the case with or without ND and SFS (dotted and thick solid horizontal lines). But double dashed lines corre-

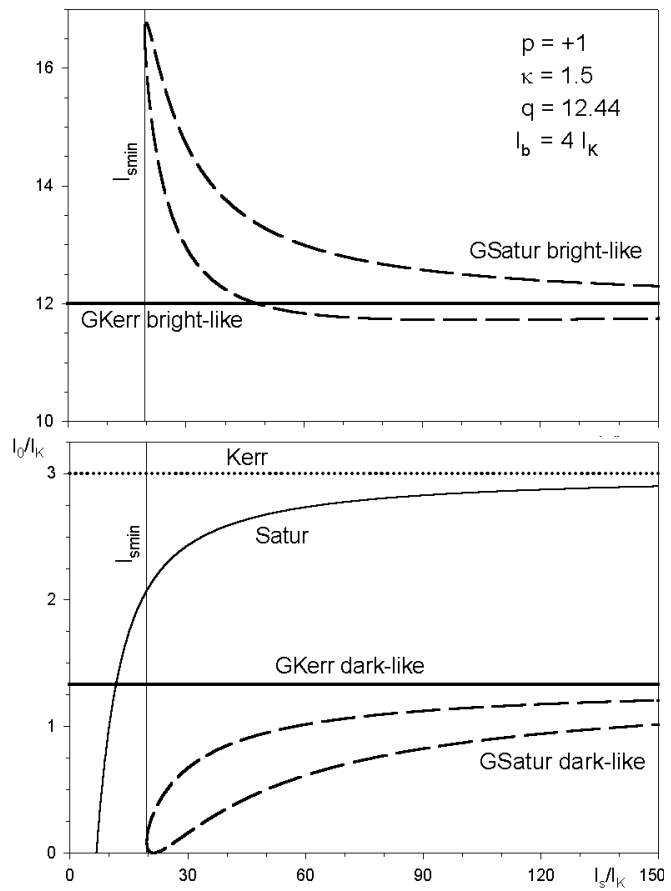

Fig. 2. Central intensity $I_{0}$ of the grey bright-like (upper part) and dark-like (lower part) solitons in different types of media versus intensity of saturation $I_{\mathrm{s}}$. Two values of $I_{0}$ for any $I_{\mathrm{s}}$ in GSatur cases determine two different soliton profiles (Fig. 1). 


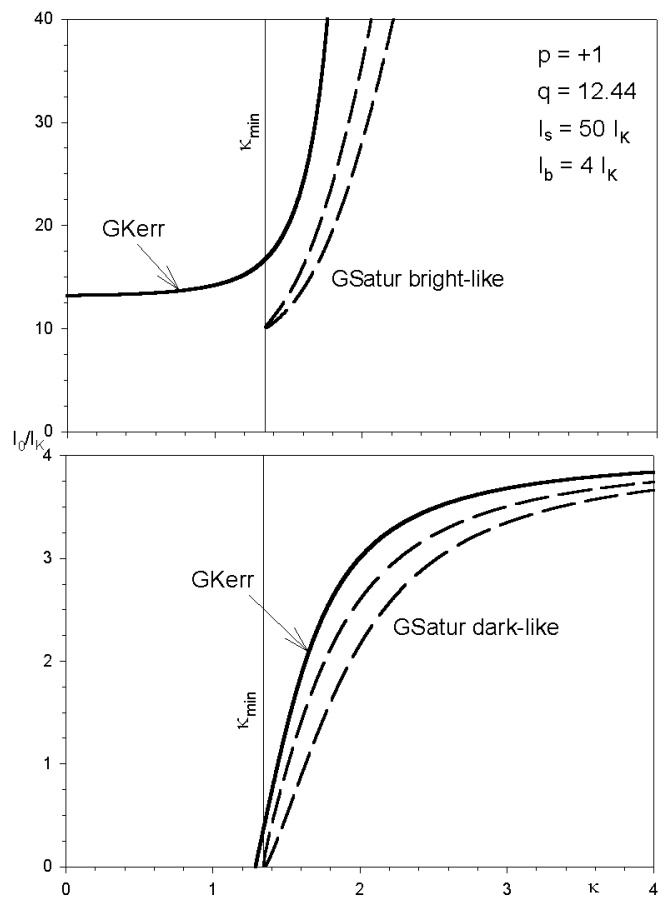

Fig. 3. Central intensity $I_{0}$ of the grey bright-like (upper part) and dark-like (lower part) solitons in generalized Kerr and generalized saturable media as functions of nonlinear corrections.

sponding to solitons in generalized saturable medium appear only for a sufficiently large saturation intensity $I_{\mathrm{s}} \geq I_{\mathrm{s} \text { min }}$. The same property reveals solitons in ordinary saturable medium, but for them $I_{\mathrm{s} \text { min }}$ is much smaller. Note that dark-like solitons decrease their height while bright-like ones increase it with decreasing $I_{\mathrm{s}}$ (this property does not apply to the regime $I_{\mathrm{s}} \approx \mathrm{I}_{\mathrm{s} \text { min }}$ in the generalized saturable case). For larger saturation levels one of the bright-like solitons has its height smaller and the other one greater than the height of the generalized Kerr soliton, while heights of both dark-like solitons are smaller than a height in generalized medium.

The lines in Fig. 3 illustrate how the central intensities depend on higher-order correction parameter $\kappa$. This time we show only lines obtained in generalized media, because the asymptotic case of the medium without ND and SFS result for $\kappa \rightarrow \infty$ and $q \rightarrow \infty$. As in Fig. 2 there is a minimum value $\kappa_{\min }$ below which solitons in generalized saturable medium cannot exist. In the generalized Kerr medium there is a similar minimum for the dark-like solitons, but for the bright-like ones the relation $I_{0}(\kappa)$ is completely different - solitons appear only for $|\kappa| \leq 2$. Therefore we can meet the situation when only dark-like, only bright-like or both solitons appear in such medium. 


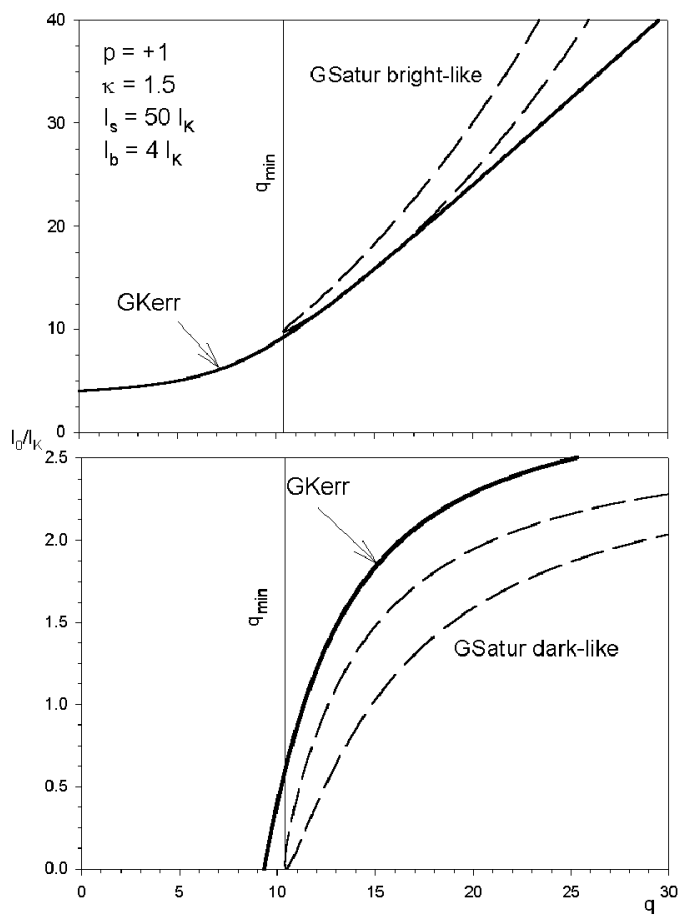

Fig. 4. Central intensity $I_{0}$ of the grey bright-like (upper part) and dark-like (lower part) solitons in generalized Kerr and generalized saturable media as functions of temporal width.

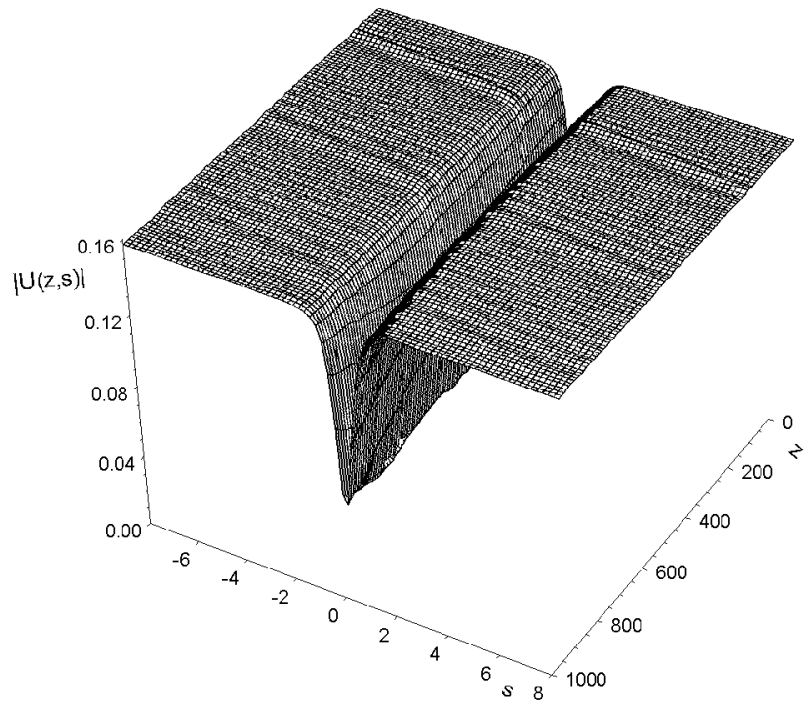

Fig. 5. Propagation of perturbed grey soliton with tuned linear chirp. $I_{0}$ increased $5 \%$, width decreased $4 \%$. 
The behavior of central intensity as a function of temporal width $q$ is similar (Fig. 4). In the generalized saturable medium we also obtain a minimum value $q_{\mathrm{min}}$. But in the generalized Kerr case one property changes - there is no maximum $q$ for the bright-like solitons. Therefore such solitons with any temporal width can exist.

For both generalized Kerr and saturable media we can write the conditions determining the discussed minimum values $I_{\mathrm{s} \text { min }}, \kappa_{\min }$, and $q_{\min }$ in the form of polynomial equations. But the order of these equations (at least for saturable case) is very high, so we can obtain such values only numerically.

Grey solitons are generally unstable. They are very sensitive with respect to phase modifications. Therefore if we modify its height or width, the obtained pulse will not propagate stably, because its phase profile is wrong. But tuning the pulse width to its changed height and applying additional linear chirp we can significantly stabilize it. In Fig. 5 we show how such modified pulse propagates over a long distance. Its central intensity and width pulsate, the background intensity diminishes and group velocity changes, but all these modifications are quite small. The presence of saturation in medium stabilizes the propagation only slightly pulsations of height and width are smaller, but changes of the other quantities remain almost the same.

\section{Summary and conclusions}

The additional terms describing nonlinear dispersion and self-frequency shift extend the class of media in which grey solitons appear. In generalized media taking these effects into account solitons can exist for both possible signs of $k_{2} / \alpha$. The class of solitons is also extended - we obtain ordinary dark-like and upturned bright-like solitons. The additional saturation of the medium causes that the central intensity and all other parameters of both dark-like and bright-like soliton can assume two possible values. All these properties can be seen in Figs. 1-4.

In generalized media even with saturation we can obtain quadratures describing both the intensity and phase profiles of grey solitons. These quadratures give analytical formulae for the square-two-level model of saturation. The simpler cases of the media without saturation or without higher-order terms also give analytical expressions for the intensity and phase profiles. The resulting intensity shapes are quite similar, however parameters of solitons depend on material parameters and are different in different media. On the other hand, the phase profiles, even when the shapes of solitons are similar, can differ quite significantly.

In generalized saturable media the possible level of saturation $I_{\mathrm{s}}$, higher-order corrections parameter $\kappa$, and temporal width parameter $q$ cannot be too small. The analogous restriction in generalized Kerr medium appears only for dark-like solitons. Generally, the presence of saturation diminishes a height of resulting solitons. 
The obtained solitons are unstable, mainly with respect to phase modifications. Nevertheless, by applying properly chosen linear chirp we can significantly stabilize them (Fig. 5).

\section{References}

[1] G.P. Agrawal, Nonlinear Fiber Optics, Academic Press, San Diego 1995, p. 28.

[2] V.B. Zakharov, A.B. Shabath, Sov. Phys. JETP 34, 62 (1972).

[3] G.P. Agrawal in: Contemporary Nonlinear Optics, Eds. G.P. Agrawal, R.W. Boyd, Academic Press, San Diego 1995, p. 41.

[4] J.N. Elgin, Opt. Lett. 17, 1409 (1992).

[5] M. Piché, J.-F. Cormier, X. Zhu, Opt. Lett. 21, 845 (1996).

[6] G. Dong, Z. Liu, Opt. Commun. 128, 8 (1996).

[7] D. Anderson, M. Lisak, Phys. Rev. A 27, 1393 (1983).

[8] R.H. Enns, S.S. Rangnekar, Phys. Rev. A 43, 4047 (1991).

[9] J.R. de Oliveira, M.A. de Moura, J.M. Hickman, A.S.L. Gomes, J. Opt. Soc. Am. B 9, 2025 (1991).

[10] J.P. Gordon, Opt. Lett. 11, 662 (1986).

[11] Y.S. Kivshar, B.A. Malomed, Opt. Lett. 18, 485 (1993).

[12] G.P. Agrawal, C. Headley, Phys. Rev. A 46, 1573 (1992).

[13] K. Hizanidis, D.J. Frantzeskakis, C. Polymilis, J. Phys. A 29, 7687 (1996).

[14] M. Gedalin, T.C. Scott, Y.B. Band, Phys. Rev. Lett. 78, 448 (1997).

[15] S. Liu, X. Liu, Phys. Lett. A 225, 67 (1997).

[16] S.L. Palacios, J.M. Fernández-Díaz, A. Guinea, J. Mod. Opt. 47, 711 (2000).

[17] S. Gatz, J. Herrmann, J. Opt. Soc. Am. B 8, 2296 (1991).

[18] U. Langbein, F. Lederer, T. Peschel, H.E. Ponath, Opt. Lett. 10, 571 (1985).

[19] W. Królikowski, X. Yang, B. Luther-Davies, J. Breslin, Opt. Commun. 105, 219 (1994).

[20] F.G. Bass, V.V. Konotop, S.A. Puzenko, Phys. Rev. A 46, 4185 (1992).

[21] H.W. Schrümann, Phys. Rev. E 54, 4312 (1996).

[22] W. Chao, Opt. Commun. 175, 239 (2000).

[23] J. Jasiński, Opt. Commun. 172, 325 (1999).

[24] J. Jasiński, Acta Phys. Pol. A 99, 77 (2001).

[25] P.F. Byrd, M.D. Friedman, Handbook of Elliptic Integrals for Engineers and Scientists, Springer-Verlag, New York 1971, p. 8, 120. 